\title{
Yang-Baxterization of Braid Group Representations
}

\author{
Y. Cheng ${ }^{1, \star}$, M. L. Ge ${ }^{1, \star \star}$, and K. Xue ${ }^{2}$ \\ 1 Institute for Theoretical Physics, State University of New York at Stony Brook, Stony Brook, \\ NY 11794-3840, USA \\ 2 Theoretical Physics Division, Nankai Institute of Mathematics, Tianjin 300071, P.R. China
}

Received May 7, 1990

\begin{abstract}
For a given braid group representation (BGR), a process of the YangBaxterization is formulated to generate solutions of the Yang-Baxter equation (YBE). When a BGR admits the Birman-Wenzl (BW) algebraic structure, this process can be explicitly passed through and two types of trigonometric solutions of YBE are generated from such a BGR. These two solutions have the essential difference to each other and both of them preserve the crossing symmetry property if the given BGR has. By taking certain reduction on the BW algebra, the rational solution is also generated. A practical condition to judge whether a BGR satisfies the BW algebra is given, from which one finds that not only the familiar BGRs of $[5,7,9]$, but also some new ones obtained recently in [12] have the BW structure. Thus they can be explicitly Yang-Baxterized to solutions of the YBE.
\end{abstract}

\section{Introduction}

It is known, nowadays, that the Yang-Baxter equation (YBE) plays a central role in the study of integrable models in statistical mechanics and quantum field theory, and is also closely related to some other fields, such as the quantum group, knot theory and conformal field theory etc., in both mathematics and physics [1-4].

Based on the theory of quantum group, or the $q$-analogues of the universal enveloping Lie algebras, Jimbo [5] constructed a family of trigonometric solutions of the YBE associated with the fundamental representations of the Kac-Moody algebras, and they possess the classical limits of Belavin and Drinfeld [6]. By letting the spectral parameter be zero (or infinity in a different choice) on the other hand, these solutions give rise to the braid group representations (BGRs)

\footnotetext{
${ }^{\star}$ Permanent address: Department of Mathematics, University of Science and Technology of China, Hefei 230026, Anhui, P.R. China

${ }^{\star \star}$ Permanent address: Theoretical Physics Division, Nankai Institute of Mathematics, Tianjin 300071, P.R. China
} 
which satisfy the Jones, or cubic reduction relations [7, 8]. The BGRs obtained in this way can also be derived directly from the quantum group of the classical simple Lie algebras [9]. Recently, some nonstandard BGRs have been found by directly solving the braid relation (i.e., the parameter independent YBE) under the constraints of the weight conservations [10-12]. We call them nonstandard BGRs because of the nonexistence of the classical limits in the sense of [9]. For some of them, the eigenvalues have the essential multiplicity two and the correspondent Markov traces cannot be defined properly in the usual way [11, 17].

The intention of this paper is as follows: starting from a given BGR, how to recover the solution of YBE which takes the given BGR as its suitable limit when the installed spectral parameter goes to zero. The process of answering this question is, now, called the Yang-Baxterization [13, 14]. We find that for a BGR $S$, the Yang-Baxterized $S$ denoted by $\check{R}(x)$ solves the YBE if and only if $S$ satisfies an identity and a sufficient condition such that $S$ satisfies this identity is that $S$ admits additional relations in the Birman-Wenzl (BW) algebra besides the braid relation.

As is known, the so-called BW algebra is a complex algebra with unit depending on two complex parameters [15]. It was designed partially to help understand Kauffman's polynomial in knot theory. In a recent paper, Jones [13] briefly gives a beautiful theorem of Yang-Baxterizing the BW algebra. In this paper, however, we would like to discuss the Yang-Baxterization prescription from a slightly different viewpoint and its further properties. We find that 1) the structure of the BW algebra exists for many of the BGRs, not only for the known standard ones of $[5,9]$ but also for the nonstandard ones of $[11,12,14]$ which are not necessarily to be related to the usual link polynomials. For later use, a practical condition to judge whether BGRs have the BW structure is given. 2) For a given BGR satisfying the BW algebra, the trigonometric Yang-Baxterization is formulated, which gives rise to two independent types of trigonometric solutions of the YBE. In particular, for the BGR of $[5,9]$ besides the solution in the form of [13], there is another one corresponding to the Kac-Moody algebras. 3) The process of Yang-Baxterization preserves the crossing symmetry property which is in the sense of $[8,16]$, i.e., if the given BGR has the crossing symmetry property, so do its Yang-Baxterized solutions of YBE. 4) If the generators and parameters of the BW algebra depend on a q-parameter, by taking a certain limit, the BW algebra reduces to the "rational" one which in turn gives rise to the rational solutions of the YBE through the rational Yang-Baxterization. We find that the BW algebras associated with all BGRs of $[5,9]$ and the nonstandard ones of $[10$, 12] have the rational reductions. Thus, the correspondent rational solutions (and furthermore the $S$-matrices) can be constructed explicitly.

\section{Sufficient Conditions for the Birman-Wenzl Algebra}

The BW algebra is generated by the unit $I$, the braid operators $S_{j}$ and the monoid operators $E_{j}$ and depends on two independent parameters $w$ and $\sigma$ [15]. Since it will be used to carry out the Yang-Baxterization in this section, we will discuss sufficient conditions for a BGR having the BW algebraic structure and give some typical examples of representations of BW algebra. Let us take the BW relations 
as follows.

$$
\begin{gathered}
S_{j}-S_{j}^{-1}=w\left(I-E_{j}\right) \\
S_{j} S_{j \pm 1} S_{j}=S_{j \pm 1} S_{j} S_{j \pm 1}, \quad S_{j} S_{k}=S_{k} S_{j}, \quad|j-k| \geq 2, \\
E_{j} E_{j \pm 1} E_{j}=E_{j}, \quad E_{j} E_{k}=E_{k} E_{j} ; \quad|j-k| \geq 2 \\
E_{j} S_{j}=S_{j} E_{j}=\sigma E_{j}, \\
S_{j \pm 1} S_{j} E_{j \pm 1}=E_{j} S_{j \pm 1} S_{j}=E_{j} E_{j \pm 1} \\
S_{j \pm 1} E_{j} S_{j \pm 1}=S_{j}^{-1} E_{j \pm 1} S_{j}^{-1} \\
E_{j \pm 1} E_{j} S_{j \pm 1}=E_{j \pm 1} S_{j}^{-1}, \quad S_{j \pm 1} E_{j} E_{j \pm 1}=S_{j}^{-1} E_{j \pm 1} \\
E_{j} S_{j \pm 1} E_{j}=\sigma^{-1} E_{j} \\
E_{j}^{2}=\left(1-\frac{\sigma-\sigma^{-1}}{w}\right) E_{j}
\end{gathered}
$$

therefore

$$
\begin{gathered}
S_{j}^{2}=w\left(S_{j}-\sigma E_{j}\right)+1, \\
S_{j}^{3}=(w+\sigma) S_{j}^{2}+(1-w \sigma) S_{j}-\sigma .
\end{gathered}
$$

If $w=q-q^{-1}$, then (2.1.11) is the following cubic reduction relation

$$
\left(S_{j}-q\right)\left(S_{j}+q^{-1}\right)\left(S_{j}-\sigma\right)=0 .
$$

In a series papers of Wadati et al. (cf. [8]), the above BW algebra is derived from the solution of YBE with the crossing symmetry property and the standard initial condition. What we will show in this section is a sufficient condition such that the BW algebra can be derived from a given BGR. We assume that the given BGR $S, S \in \operatorname{End}\left(C^{N} \otimes C^{N}\right)$ satisfies the cubic reduction relation (2.2) and

1. the charge (or spin) conservation condition:

$$
S_{c d}^{a b} \neq 0 \text { only for } a+b=c+d,
$$

2. the invariant conditions under the CPT transformation:

$$
S_{c d}^{a b}=S_{d^{\prime} c^{\prime}}^{b^{\prime} a^{\prime}}
$$

where

$$
S=\sum S_{c d}^{a b}\left(e_{a c} \otimes e_{b d}\right)
$$

and $e_{a b}$ is the $N \times N$ matrix with $\left(e_{a b}\right)_{i j}=\delta(a, i) \delta(b, j), \delta(a, b)=1$ for $a=$ $b, \delta(a, b)=0$ for $a \neq b$. The indices run over $1,2, \ldots, N$ and the notation $a^{\prime}$ is understood as the charge conjugation, i.e. $a^{\prime}=N+1-a$. Define a matrix $E \in \operatorname{End}\left(C^{N} \otimes C^{N}\right)$ as follows:

$$
E=I-\frac{1}{w}\left(S-S^{-1}\right) \equiv \sum E_{c d}^{a b}\left(e_{a c} \otimes e_{b d}\right),
$$

then the sufficient conditions such that

$$
\begin{gathered}
S_{j}=I \otimes \cdots \otimes S \otimes \cdots \otimes I, \\
E_{j}=I \otimes \cdots \otimes E \otimes \cdots \otimes I,
\end{gathered}
$$


and $w=q-q^{-1}, \sigma$ give rise to the representation of the BW algebra are

$\begin{aligned} \text { 1) } E_{c d}^{a b} & =r(a) r(c) \delta\left(a, b^{\prime}\right) \delta\left(c, d^{\prime}\right), \\ \text { 2) } r(a) r\left(a^{\prime}\right) & =1, \\ \text { 3) } \sum_{b} S_{a b}^{a b} r^{2}(b) & \left.=\sigma^{-1} \text { (independent of } a\right),\end{aligned}$

where $I$ is the $N \times N$ unit matrix. The condition (2.9) implies that $E$ is a block diagonal matrix with only the central $N \times N$ block being nontrivial. To check the conditions are sufficient to imply the BW algebra, one first uses (2.9)-(2.11) to get (2.1.3) and (2.1.8) simply by the direct calculation, then using the cubic reduction relation (2.2) and (2.6), the relation (2.1.4) can be obtained. As a result

$$
E^{2}=\frac{1}{w \sigma}\left(1+w S-S^{2}\right) E=\left(1-\frac{\sigma-\sigma^{-1}}{w}\right) E
$$

which implies (2.1.9). The other relations in (2.1) can also be derived from the well checked results. The following shows some examples of the BW algebra representations.

Example 2.1. For the standard BGR of $C_{n}$ and $D_{n}$.

They are obtained from $[5,7,9]$ and have the form

$$
\begin{aligned}
S= & q \sum e_{i i} \otimes e_{i i}+\sum_{i \neq j, j^{\prime}} e_{i j} \otimes e_{j i}+q^{-1} \sum e_{i i^{\prime}} \otimes e_{i^{\prime} i} \\
& +w \sum_{i<j}\left(e_{i i} \otimes e_{j j}-\varepsilon \varepsilon_{i} \varepsilon_{j} q^{\bar{i}-\bar{j}} e_{j^{\prime} i} \otimes e_{j i^{\prime}}\right),
\end{aligned}
$$

where the indices run from 1 to $N=2 n, i^{\prime}=N+1-i, \varepsilon_{i}=1$ for $i<i^{\prime}, \varepsilon_{i}=\varepsilon$ for $i>i^{\prime}, \varepsilon=-1$ for $C_{n}, \varepsilon=1$ for $D_{n}$ and

$$
\bar{i}= \begin{cases}i+\frac{1}{2} \varepsilon & i<i^{\prime} \\ i-\frac{1}{2} \varepsilon & i>i^{\prime}\end{cases}
$$

$S$ in (2.13) satisfies the cubic reduction relation [5, 9]

$$
(S-q)\left(S+q^{-1}\right)\left(S-\varepsilon q^{-N+\varepsilon}\right)=0 .
$$

The matrix $E$ defined in (2.6) in this case has the form [5]

$$
E=-\sum \varepsilon \varepsilon_{i} \varepsilon_{j} q^{\bar{i}-\bar{j}}\left(e_{j^{\prime} i} \otimes e_{j i^{\prime}}\right) .
$$

By calculations, we have

$$
\begin{gathered}
S_{c d}^{a b}=q \delta(a, b)+w \theta(a, b)\left[1-\varepsilon_{a} \varepsilon_{b} q^{\bar{a}-\bar{b}} \delta\left(a, b^{\prime}\right)\right] \\
E_{c d}^{a b}=-\varepsilon \varepsilon_{c} \varepsilon_{a^{\prime}} q^{\bar{c}-\bar{a}^{\prime}} \delta\left(a, b^{\prime}\right) \delta\left(c, d^{\prime}\right) \\
=r(a) r(c) \delta\left(a, b^{\prime}\right) \delta\left(c, d^{\prime}\right)
\end{gathered}
$$

with $\theta(a, b)=1$ for $a<b, \theta(a, b)=0$ for $a \geq b$ and

$$
\begin{aligned}
r(a) & =\exp \left[\frac{i \pi}{4}(1-\varepsilon)\right] \varepsilon_{a} q^{\bar{a}-\frac{N+1}{2}}, \\
a & =1.2, \ldots, N,
\end{aligned}
$$


here we have used $\bar{a}^{\prime}=N+1-\bar{a}$ and $\varepsilon_{a^{\prime}}=\varepsilon \varepsilon_{a}$. One can then check that $S$ and $E$ in (2.13) and (2.16) satisfy the conditions (2.9)-(2.11) with $\sigma=\varepsilon q^{-N+\varepsilon}$, thus (2.7) and (2.8) satisfy the BW relations. This fact has been indicated in [5, 9].

Example 2.2. For nonstandard BGRs of $C_{2}$ and $D_{2}$.

These BGRs are obtained by directly solving the braid relation under the constraint of the weight conservations [10], they are in the form of block diagonal matrix.

$$
S=\text { block diag. }\left(A_{1}, A_{2}, A_{3}, A_{4}, A_{3}, A_{2}, A_{1}\right)
$$

with the block submatrices [11]

$$
\begin{aligned}
& A_{1}=q, A_{2}=\left[\begin{array}{ll}
0 & 1 \\
1 & w
\end{array}\right], \\
& A_{3}=\left[\begin{array}{ccc}
0 & 0 & 1 \\
0 & -q^{-1} & 0 \\
1 & 0 & w
\end{array}\right],
\end{aligned}
$$

and

$$
A_{4}=\left[\begin{array}{cccc}
0 & 0 & 0 & q^{-1} \\
0 & 0 & -q & -i w \\
0 & -q & \left(1+q^{2}\right) w & i q^{2} w \\
q^{-1} & -i w & i q^{2} w & \left(1-q^{2}\right) w
\end{array}\right]
$$

for $C_{2}$, and

$$
\left[\begin{array}{cccc}
0 & 0 & 0 & q^{-1} \\
0 & 0 & -q & -i w \\
0 & -q & 0 & -i w \\
q^{-1} & -i w & -i w & 2 w
\end{array}\right]
$$

for $D_{2}$. These BGRs also satisfy the cubic reduction relation

$$
(S-q)^{2}\left(S+q^{-1}\right)=0
$$

for $C_{2}$, and

$$
(S-q)\left(S+q^{-1}\right)^{2}=0
$$

for $D_{2}$, but in both cases, there are only two distinct eigenvalues with one having the essential multiplicity two. The matrix $E$ defined in (2.6) for the present example has exactly the form (2.9) with $N=4$ and

$$
r(1)=q^{-1}, \quad r(2)=i q^{-1}, \quad r(3)=-i q, \quad r(4)=q
$$

for $C_{2}$ and

$$
r(1)=i, \quad r(2)=1, \quad r(3)=1, \quad r(4)=-i
$$

for $D_{2}$. One can check that $S$ in (2.20)-(2.23) and $E$ in (2.6) with (2.26) and (2.27) respectively, satisfy (2.9)-(2.11) with $\sigma=q$ for $C_{2}$ and $\sigma=-q^{-1}$ for $D_{2}$. Thus in both cases of $C_{2}$ and $D_{2}$, the BGRs have the BW algebraic structures.

Remarks. 1) The standard BGR of $B_{n}$ of [5,9] and the nonstandard ones of $B_{n}$, $C_{n}$ and $D_{n}$ given in [12] have also the BW relations, however, to save space, we omit them. 2) The BW algebra was designed to help understand Kauffman's link polynomial [15]. The example 2 here shows that there exist BGRs having BW algebraic structure but the usual scheme of constructing the link polynomial is 
not valid for them, since the correspondent Markov traces cannot be defined in the usual way [17].

In most practical cases, the parameters $w$ in the BW algebra have the form $w=q-q^{-1}$ and $\sigma$ is proportional to the (positive, or negative) power of $q$. Thus we transform $w, \sigma$ formally by

$$
w=q-q^{-1}, \quad \sigma= \pm q^{s}
$$

to other parameters $q$ and $s$, and assume the generators of the BW algebra depend on $q$ without singularity around $q=1$. By defining

$$
T_{j}=\lim _{q \rightarrow 1} S_{j}, \quad F_{j}=\lim _{q \rightarrow 1} E_{j},
$$

we find

$$
T_{j}^{2}=1, \quad F_{j}=1-\frac{1}{2} d\left(S_{j}-S_{j}^{-1}\right) /\left.d q\right|_{q=1},
$$

from (2.1.10) and (2.1.1). The other relations in (2.1) are reduced to

$$
\begin{gathered}
T_{j} T_{J \pm 1} T_{j}=T_{j \pm 1} T_{j} T_{j \pm 1}, T_{i} T_{j}=T_{j} T_{l}, \quad|i-j| \geq 2, \\
F_{j} F_{j \pm 1} F_{j}=F_{j}, F_{l} F_{j}=F_{j} F_{i}, \quad|i-j| \geq 2, \\
F_{J} T_{j}=T_{j} F_{j}= \pm F_{j} \\
T_{j \pm 1} T_{j} F_{J \pm 1}=F_{j} T_{j \pm 1} T_{j}=F_{j} F_{j \pm 1} \\
T_{j \pm 1} F_{j} T_{j \pm 1}=T_{j} F_{j \pm 1} T_{j} \\
F_{j \pm 1} F_{j} T_{J \pm 1}=F_{J \pm 1} T_{j}, T_{j \pm 1} F_{j} F_{j \pm 1}=T_{j} F_{j \pm 1} \\
F_{j} T_{j \pm 1} F_{j}= \pm F_{j}, F_{j}^{2}=(1 \mp s) F_{j} .
\end{gathered}
$$

It is easy to see that the examples of representations of the BW algebra in this section admit this reduction and the "parameter" $s$ in each case only takes the fixed value of integer. Some other reduction of the BW algebra by taking special value of one parameter and its mathematical significiences are discussed in [18].

\section{The Trigonometric Yang-Baxterization}

In this section, we start from the given BGR to generate the trigonometric solution of the YBE

$$
\check{R}_{1}(x) \check{R}_{2}(x y) \check{R}_{1}(y)=\check{R}_{2}(y) \check{R}_{1}(x y) \check{R}_{2}(x)
$$

through the (trigonometric) Yang-Baxterization. The theorem of Jones [13] indicates that the BGR satisfying the BW algebra can be Yang-Baxterized to the solution of YBE (3.1). What we shall do here is to formulate this process from other viewpoint and discuss the properties when the Yang-Baxterization is carried out.

We will restrict our discussion on the BGR satisfying the following cubic reduction relation

$$
\left(S-\lambda_{1}\right)\left(S-\lambda_{2}\right)\left(S-\lambda_{3}\right)=0
$$

where $\lambda_{i}, i=1,2,3$ are eigenvalues of $S$.

According to the analysis of [14], we assume that the solutions of YBE is in the form

$$
\check{R}(x)=A(x) S+B(x) I+C(x) S^{-1} .
$$


By the requirement that (3.3) satisfies

1) the boundary condition: $\check{R}(0) \propto S$,

2) the initial condition: $\quad \check{R}(1) \propto I$,

3) the unitarity condition: $\check{R}(x) \check{R}\left(x^{-1}\right)=f(x) I$,

for some function $f(x)$, then the coefficients $A(x), B(x)$ and $C(x)$ in (3.3) can be chosen in the following ways:

$$
\text { Case (a), } \begin{aligned}
A(x) & =-\lambda_{3}^{-1}(x-1), C(x)=\lambda_{1} x(x-1), \\
B(x) & =\left(1+\frac{\lambda_{1}}{\lambda_{2}}+\frac{\lambda_{1}}{\lambda_{3}}+\frac{\lambda_{2}}{\lambda_{3}}\right) x
\end{aligned}
$$

Case (b), $\quad A(x)=-\lambda_{3}^{-1}(x-1), C(x)=\lambda_{2} x(x-1)$,

$$
B(x)=\left(1+\frac{\lambda_{2}}{\lambda_{1}}+\frac{\lambda_{2}}{\lambda_{3}}+\frac{\lambda_{1}}{\lambda_{3}}\right) x
$$

Case (c), $\quad A(x)=-\lambda_{2}^{-1}(x-1), C(x)=\lambda_{1} x(x-1)$,

$$
B(x)=\left(1+\frac{\lambda_{1}}{\lambda_{3}}+\frac{\lambda_{1}}{\lambda_{2}}+\frac{\lambda_{3}}{\lambda_{2}}\right) x .
$$

Notice that the functions $A(x), B(x)$ and $C(x)$ in case (b) and case (c) can be obtained respectively from case (a) under the permutation

$$
\left(\lambda_{1}, \lambda_{2}, \lambda_{3}\right) \rightarrow\left(\lambda_{2}, \lambda_{1}, \lambda_{3}\right)
$$

for case (b) and

$$
\left(\lambda_{1}, \lambda_{2}, \lambda_{3}\right) \rightarrow\left(\lambda_{1}, \lambda_{3}, \lambda_{2}\right)
$$

for case (c).

Corresponding to each case in (3.7), (3.8), and (3.9), $\check{R}(x)$ in (3.3) solves the YBE (3.1) if and only if the BGR $S$ satisfies the following identity [14]

$$
f_{3}^{+} \theta_{3}^{+}+f_{3}^{-} \theta_{3}^{-}+f_{2} \theta_{2}+f_{1}^{+} \theta_{1}^{+}+f_{1}^{-} \theta_{1}^{-}=0,
$$

where

$$
\begin{aligned}
\theta_{3}^{ \pm} & =S_{1}^{ \pm 1} S_{2}^{\mp 1} S_{1}^{ \pm 1}-S_{2}^{ \pm 1} S_{1}^{\mp 1} S_{2}^{ \pm 1} \\
\theta_{2} & =S_{1} S_{2}^{-1}-S_{2} S_{1}^{-1}+S_{2}^{-1} S_{1}-S_{1}^{-1} S_{2} \\
\theta_{1}^{ \pm} & =S_{1}^{ \pm 1}-S_{2}^{ \pm 1}
\end{aligned}
$$

and $f_{3}^{ \pm}, f_{2}, f_{1}^{ \pm}$are given by

$$
\begin{aligned}
f_{3}^{+} & =\frac{\lambda_{1}}{\lambda_{3}^{2}}, \quad f_{3}^{-}=-\frac{\lambda_{1}^{2}}{\lambda_{3}}, \\
f_{2} & =-\frac{\lambda_{1}}{\lambda_{3}}\left(1+\frac{\lambda_{1}}{\lambda_{2}}+\frac{\lambda_{2}}{\lambda_{3}}+\frac{\lambda_{1}}{\lambda_{3}}\right), \\
f_{1}^{ \pm} & =\mp \lambda_{2}^{\mp 1} f_{2},
\end{aligned}
$$

for case (a). For case (b) and case (c), they can be written down from (3.14) by taking the transformation (3.10) and (3.11) respectively.

The problem of demonstration that $\check{R}(x)$ in (3.3) solves the YBE now becomes to check whether the given BGR $S$ satisfies the identity (3.12) for some of the 
cases. We find that a sufficient condition for $S$ satisfying (3.12) is that $S$ admits the BW algebraic structure. Without losing the generality, we assume

$$
\sigma=\lambda_{3}, \quad w=\lambda_{1}+\lambda_{2}, \quad \lambda_{1} \lambda_{2}=-1,
$$

$\left(\lambda_{1} \lambda_{2}=-1\right.$ can be realized by taking a normalization of $\left.S\right)$ and $S$ and $E$ defined through (2.1.1) satisfy the BW relation (2.1). Then $S$ satisfies (3.12) for case (a) and case (b), therefore we have two solutions of the YBE (3.1) which are in the form of (3.3) with coefficients given in (3.7) and (3.8) respectively.

In terms of the generators of the BW algebra (2.1), the solutions in (3.3) can be expressed as

$$
\check{R}_{\alpha}(x)=a_{\alpha}(x) S+b_{\alpha}(x) E+c_{\alpha}(x) I, \quad \alpha=a, b,
$$

with

$$
\begin{aligned}
& a_{\alpha}(x)=(x-1)\left(x-x_{\alpha}\right), \quad b_{\alpha}(x)=w x(x-1), \\
& c_{\alpha}(x)=-w x\left(x-x_{\alpha}\right), \quad \alpha=a, b,
\end{aligned}
$$

and $x_{a}, x_{b}$ are fixed points given by

$$
x_{a}=-q \sigma^{-1}, \quad x_{b}=q^{-1} \sigma^{-1},
$$

here we have set $\lambda_{1}=q, \lambda_{2}=-q^{-1}$ since $\lambda_{1} \lambda_{2}=-1$ was assumed before.

One can also check directly that both $\check{R}_{a}(x)$ and $\check{R}_{b}(x)$ satisfy the conditions (3.4), (3.5) and (3.6), and solve the YBE (3.1). For instance, to check that $\check{R}_{\alpha}(x)$, $\alpha=a, b$ solve (3.1), one substitutes (3.16) into (3.1) and considers the difference denoted by $\phi(x, y)$ between two sides of (3.1). For a fixed value of $y, \phi(x, y)$ is a fourth order polynomial in $x$, it is sufficient to check that $\phi(x, y)$ vanishes at five points. At $x=1, x=y^{-1}, \phi(x, y)=0$ trivially. At $x=0$ (similarly at $x=\infty$ ), $\phi(x, y)=0$ after using the BW relations (2.1.2), (2.1.3) and (2.1.5). At $x=x_{\alpha}$

$$
\phi\left(x_{\alpha}, y\right)=b\left(x_{\alpha}\right)\left[E_{1} \check{R}_{2}\left(x_{\alpha} y\right) \check{R}_{1}(y)-\check{R}_{2}(y) \check{R}_{1}\left(x_{\alpha} y\right) E_{2}\right]
$$

which is the fourth order polynomial in $y$. In the similar way, (3.19) vanishes at $y=0,1, x_{\alpha}, x_{\alpha}^{-1}$ and $y=\infty$.

The conclusion, now, becomes clear that each BGR satisfying the BW algebra (2.1) gives rise to two solutions of YBE [i.e., (3.3) for case (a) and case (b), or $(3.16)]$. In particular, the first solution $\check{R}_{a}(x)$ coincides with that in Jones theorem [13] by a suitable normalization. One explanation of this "one to two" correspondence is in the sense that the BW algebra (2.1) admits the invariant transformation of (3.10) (i.e., $q \rightarrow-q^{-1}, \sigma \rightarrow \sigma$ ) under the assumption (3.15), however, it is by no means that the result is trivial. For the standard BGRs of $B_{n}$ and $D_{n}$ in (2.13), the correspondent two solutions in (3.16) are Jimbo's [5] solutions which are respectively associated with the quantum group picture of $B_{n}^{(1)}$ and $D_{n}^{(1)}$ (for $\alpha=a$ ) and the Kac-Moody types $A_{2 n}^{(2)}$ and $A_{2 n-1}^{(2)}$ (for $\alpha=b$ ). Corresponding to the BGR of $C_{n}$ in (2.13), $\check{R}_{b}(x)$ is Jimbo's of $C_{n}^{(1)}$, and $\check{R}_{a}(x)$ is a new one for which the Kac-Moody quantum group structure is not clear yet. For the nonstandard case we also have "usual" solutions and "twisted" solutions according to case (a) and case (b).

It should be emphasized that the BW algebra is only the sufficient condition for the Yang-Baxterization since there exist some BGRs having no BW structure but satisfy the identity (3.12) [19] and so for these BGRs, one can still have the solution of YBE in the form of (3.3). 
Let us discuss some other properties of the solutions of YBE in (3.16).

Proposition 1. The solutions in (3.16) have the following fractional form:

$$
\begin{aligned}
& \check{R}_{a}(x)=f_{a}(x) \frac{q x+S}{q+x S}, \quad x \neq-1, \\
& \check{R}_{b}(x)=f_{b}(x) \frac{-q^{-1} x+S}{-q^{-1}+x S}, \quad x \neq-1,
\end{aligned}
$$

where

$$
f_{a}(x)=q^{-1}\left(x-q^{2}\right)\left(x-x_{a}\right), \quad f_{b}(x)=q\left(x-q^{-2}\right)\left(x-x_{b}\right) .
$$

The proof is simply the calculation by multiplying the both sides of (3.20) with $(q+x S)$ and (3.21) with $\left(-q^{-1}+x S\right)$ and using the BW relations (2.1.4), (2.1.9), and (2.1.10).

Proposition 2. Suppose $S$ and satisfy the BW algebra (2.1) and $E$ has the form (2.9) with $r(a)$ satisfy (2.10). If $R=P S$ satisfies the crossing symmetry property $[8,16]$

$$
R=C_{2}\left(R^{-1}\right)^{t_{2}} C_{2}^{-1}
$$

with

$$
\begin{aligned}
C_{2} & =(I \otimes C), \quad C \text { being an } N \times N \text { matrix } \\
C & =\left(C_{a b}\right), \quad C_{a b}=r(a) \delta\left(a, b^{\prime}\right),
\end{aligned}
$$

then

$$
\begin{aligned}
& R_{\alpha}(x)=P \check{R}_{\alpha}(x) \text { also satisfies the crossing symmetry property } \\
& R_{\alpha}(x)=F_{\alpha}(x) C_{2}\left[P R_{\alpha}\left(x_{\alpha} x^{-1}\right) P\right]^{t_{2}} C_{2}^{-1}
\end{aligned}
$$

with $F_{\alpha}(x)=x_{\alpha}^{-1} x^{2}, \alpha=a, b$. Here $P$ is the permutation operator: $P(x \otimes y)=y \otimes x$ for any $x \otimes y \in C^{N} \otimes C^{N}$, " $t_{2}$ " means the transposition acting over the second space when the matrix belongs to $\operatorname{End}\left(C^{N} \otimes C^{N}\right)$.

Proof. First of all, according to the assumption, one calculates that

$$
\begin{gathered}
P E=C_{2} P^{t_{2}} C_{2}^{-1}, \\
C_{2}(E P)^{t_{2}} C_{2}^{-1}=P .
\end{gathered}
$$

Then using $S^{-1}$ instead of $S$ in (3.16) through (2.1.1) we find

$$
\check{R}_{\alpha}\left(x_{\alpha} x^{-1}\right)=F_{\alpha}^{-1}(x)\left[a_{\alpha}(x) S^{-1}+c_{\alpha}(x) E+b_{\alpha}(x) I\right]
$$

with $F_{\alpha}(x)=x_{\alpha}^{-1} x^{2}$, so

$$
R_{\alpha}\left(x_{\alpha} x^{-1}\right)=F_{\alpha}^{-1}(x)\left[a_{\alpha}(x) P R^{-1} P+c_{\alpha}(x) P E+b_{\alpha}(x) P\right] .
$$

This immediately implies (3.25) because of (3.23), (3.26), and (3.27).

Proposition 2 indicates that the Yang-Baxterization procedure preserves the crossing symmetry property. The crossing point of the solution (3.16) in each case $\alpha=a, b$ is $x_{a}$ and $x_{b}$ respectively. The monoid operator $E$ in the $\mathrm{BW}$ algebra is proportional to the value of the Yang-Baxterized $\check{R}_{\alpha}(x)$ at the crossing point $x=x_{\alpha}, \alpha=a$ and $b$ respectively. Note that in [8], starting from the solution of YBE satisfying the crossing symmetry property, the representation of the BW algebra is derived with the monoid operator being the value of the given solution of YBE at the crossing point. Our Proposition 2 gives, in some sense, an invertible process of the result of [8]. 


\section{Rational Yang-Baxterization}

Let us start from the reduced BW algebra (2.30) to generate the rational solution of the YBE

$$
\check{R}_{1}(u) \check{R}_{2}\left(u+u^{\prime}\right) \check{R}_{1}\left(u^{\prime}\right)=\check{R}_{2}\left(u^{\prime}\right) \check{R}_{1}\left(u+u^{\prime}\right) \check{R}_{2}(u) .
$$

Similar to the discussion of the trigonometric case, we suppose that the solution of (4.1) has the form

$$
\check{R}(u)=a(u) T+b(u) F+c(u) I,
$$

where $T$ and $E$ are $N^{2} \times N^{2}$ matrices and generate the reduced BW algebra (2.30) through

$$
\begin{aligned}
& T_{j}=I \otimes \cdots \otimes T \otimes \cdots \otimes I, \\
& F_{j}=I \otimes \cdots \otimes F \otimes \cdots \otimes I,
\end{aligned}
$$

and $I$ is the $N \times N$ unit matrix. By requiring that

$$
\begin{gathered}
\check{R}(0) \propto I, \\
\check{R}(\varrho) \propto F, \\
\frac{\check{R}(u)}{a(u)} \rightarrow T \text { as } u \rightarrow \infty, \\
\check{R}(u) \check{R}(-u)=f(u) I,
\end{gathered}
$$

for some point $Q$ and function $f(u)$. The simple choice of the coefficients of $\check{R}(u)$ in (4.2) such that it satisfies the first three conditions (4.5), (4.6) and (4.7) is

$$
a(u)=u(u-\varrho), \quad b(u)=b_{0} u, \quad c(u)=c_{0}(u-\varrho) .
$$

Then from the unitarity condition (4.8) and use of the relations (2.30.1), (2.30.4) and (2.30.9), we find functions in (4.9) must satisfy

$$
\begin{aligned}
f(u) I= & {[a(u) a(-u)+c(u) c(-u)] I } \\
& +[a(u) c(-u)+a(-u) c(u)] T \\
& +\{[(1 \mp s) b(u) \mp a(u)+c(u)] b(-u)+[ \pm a(-u)+c(-u)] b(u)\} F .
\end{aligned}
$$

By comparing the coefficients of $I, T, F$, we have

$$
\begin{aligned}
& \pm 2 \varrho=2 c_{0}+(1 \mp s) b_{0}, \\
& f(u)=\left(u^{2}-\varrho^{2}\right)\left(u^{2}-c_{0}^{2}\right),
\end{aligned}
$$

namely, when the coefficients in (4.2) are given by (4.9) with the constraint (4.11) on the unknown quantities $\varrho, b_{0}$ and $c_{0}$, then $\check{R}(u)$ in (4.2) satisfies all conditions (4.5)-(4.8). Unlike the case of trigonometric Yang-Baxterization, the unknown quantities cannot be fixed in the present case only by the conditions (4.5)-(4.8). With these unknown quantities satisfying the constraint (4.11), we substitute (4.2) into the YBE (4.1) and denote by $\phi\left(u, u^{\prime}\right)$ as the difference between two sides of (4.1). For the fixed $u^{\prime}, \phi\left(u, u^{\prime}\right)$ is a fourth order polynomial in $u$. It is easy to check that the coefficient of the leading terms of $\phi\left(u, u^{\prime}\right)$ is zero. At $u=0, u=-u^{\prime}$, $\phi\left(u, u^{\prime}\right)$ vanishes trivially, At $u=\varrho$

$$
\phi\left(\varrho, u^{\prime}\right)=b(\varrho)\left[F_{1} \check{R}_{2}\left(\varrho+u^{\prime}\right) \check{R}_{1}\left(u^{\prime}\right)-\check{R}_{2}\left(u^{\prime}\right) \check{R}_{1}\left(u^{\prime}+\varrho\right) F_{2}\right],
$$


and at $u=-u^{\prime}+\varrho$

$$
\phi\left(-u^{\prime}+\varrho\right)=b(\varrho)\left[\check{R}_{1}\left(\varrho-u^{\prime}\right) F_{2} \check{R}_{1}\left(u^{\prime}\right)-\check{R}_{2}\left(u^{\prime}\right) F_{1} \check{R}_{2}\left(\varrho-u^{\prime}\right)\right] .
$$

For (4.13), we have

$$
\begin{aligned}
\phi\left(\varrho, u^{\prime}\right)= & b(\varrho)\left\{\left[\mp a\left(\varrho+u^{\prime}\right) b\left(u^{\prime}\right)+b\left(\varrho+u^{\prime}\right) b\left(u^{\prime}\right)+c\left(\varrho+u^{\prime}\right) c\left(u^{\prime}\right)\right.\right. \\
& \left. \pm c\left(\varrho+u^{\prime}\right) a\left(u^{\prime}\right)-(1 \mp s) c\left(\varrho+u^{\prime}\right) b\left(u^{\prime}\right)\right] F_{1} \\
& \left.+\left[a\left(\varrho+u^{\prime}\right) c\left(u^{\prime}\right)-b\left(\varrho+u^{\prime}\right) a\left(u^{\prime}\right)\right] F_{1} T_{2}\right\}
\end{aligned}
$$

from which, one finds that the coefficients of $F_{1}$ and $F_{1} T_{2}$ are zero only when $\varrho$, $b_{0}, c_{0}$ satisfy (4.6) and $c_{0}=-b_{0}$. Thus we have

$$
c_{0}=-b_{0} \equiv \eta, \varrho_{ \pm}=\frac{s \pm 1}{2} \eta,
$$

where $s$ is in (2.30), $\eta$ is a free parameter and " \pm " corresponds to the sign in (2.28) and (2.30). Similarly we find (4.14) to be zero if $\varrho, c_{0}, b_{0}$ are given by (4.16). Therefore the rational of the YBE (4.1) is constructed, which is

$$
\check{R}_{ \pm}(u)=u\left(u-\varrho_{ \pm}\right) T-\eta u F+\eta\left(u-\varrho_{ \pm}\right) I,
$$

with $\varrho_{ \pm}$given in (4.16). We emphasize that " \pm " in (4.17) correspond to the sign in (2.28), namely, when a BGR satisfying the BW algebra (2.1) is given, then the sign in (2.28) is fixed and the reduced BW (2.30) gives rise to the rational solution of YBE in (4.17) with the same sign. The relation of the rational solution (4.17) and the trigonometric ones in (3.16) is as follows, by letting

$$
x=\exp (h u), \quad q=\exp \left(-\frac{h \eta}{2}\right),
$$

in the trigonometric solutions (3.16), as $h \rightarrow 0$, one of the solutions in (3.16) takes the rational one (4.17) as its limit while another one has no limit. More precisely, if (2.28) takes the sign "+" (respectively “-"), $\check{R}_{+}(u)$ (respectively $\check{R}_{-}(u)$ ) in (4.17) is the limit of $\check{R}_{b}(x)$ (respectively $\check{R}_{a}(x)$ ) and $\check{R}_{a}(x)$ (respectively $\check{R}_{b}(x)$ ) has no limit at the same time.

By a normalization and using $u$ to replace $u \eta^{-1}$, (4.17) becomes

$$
\check{R}_{ \pm}(u)=I+u T-u\left(u-\frac{s \pm 1}{2}\right)^{-1} F .
$$

As is known, the matrix $T$ in the representation of the reduced BW algebra (2.30) satisfies $T^{2}=I$, it is an analogue of the permutation operator $P$ and in some cases $T=P$ exactly. When $T=P$, the first two terms together are the well-known $S$-matrix of Yang [1, 20]. Thus the rational solution of YBE in (4.19) is a generalized version of the $S$-matrix of Yang and Zamolodchikov's factorization constraints [21].

Let us see some examples of the rational solution of YBE (4.1).

Example 4.1. Consider the BGRs of $C_{n}$ and $D_{n}$ in (2.13) and $E$ in (2.16). The parameters of the correspondent BW algebra are $w=q-q^{-1}$ and $\sigma=\varepsilon q^{-N+\varepsilon}$. As $q \rightarrow 1$, the BW relations in (2.1) are reduced to (2.30) with negative sign for $C_{n}$ and positive sign for $D_{n}, s=-N+\varepsilon$ and

$$
T=P, F=-\sum \varepsilon \varepsilon_{i} \varepsilon_{j}\left(e_{j^{\prime} i} \otimes e_{j i^{\prime}}\right) .
$$


Thus the rational solutions are

$$
\check{R}_{\varepsilon}(u)=I+u P-u\left(u+\frac{N}{2}-\varepsilon\right)^{-1} F,
$$

where $\varepsilon=-1$ for $C_{n}$ and $\varepsilon=1$ for $D_{n}$.

Example 4.2. Corresponding to the nonstandard BGRs of $C_{2}$ and $D_{2}$ and their representations of the BW algebra in Example 2.2 of Sect. 2. The reduced BW relations in (2.30) have the positive sign and $s=1$ for $C_{2}$, and negative sign and $s=-1$ for $D_{2}$. The rational solutions of YBE (4.1) are

$$
\check{R}_{\varepsilon}(u)=I+u T-u(u-\varepsilon)^{-1} F_{\varepsilon},
$$

with $\varepsilon=1$ for $C_{2}$ and $\varepsilon=-1$ for $D_{2}$ in this case. The matrix $T$ is

$$
T=\text { block diag }\left(T_{1}, T_{2}, T_{3}, T_{4}, T_{3}, T_{2}, T_{1}\right),
$$

with

$$
\begin{aligned}
& T_{1}=1, \\
& T_{2}=\operatorname{anti} \operatorname{diag}(1,1), \\
& T_{3}=\operatorname{anti} \operatorname{diag}(1,-1,1), \\
& T_{4}=\text { anti diag }(1,-1,-1,1),
\end{aligned}
$$

and the matrix $F_{\varepsilon}$ is also a $16 \times 16$ block diagonal matrix, but only the central $4 \times 4$ block is nonzero. This central $\left(F_{\varepsilon}\right)_{4}$ is given by

$$
\left(F_{\varepsilon}\right)_{4}=\left[\begin{array}{cccc}
\varepsilon & i \varepsilon & -i & 1 \\
i \varepsilon & -\varepsilon & 1 & i \\
-i & 1 & -\varepsilon & -i \varepsilon \\
1 & i & -i \varepsilon & \varepsilon
\end{array}\right]
$$

with $\varepsilon=1$ for $C_{2}$ and $\varepsilon=-1$ for $D_{2}$ in the present example.

Using (4.17) we can determine the corresponding $S$-matrices up to the CDD poles [21].

\section{Conclusion and Discussion}

In this paper, starting from the given BGR $S$ satisfying the cubic reduction relation, the process of the Yang-Baxterization is formulated to generate solutions of the YBE. By the assumption that $\check{R}(x)$ has the form in (3.3), the conditions (i.e., the boundary, initial and unitarity conditions) (3.4), (3.5) and (3.6) immediately give rise to three kinds of explicit expressions of coefficients $A(x), B(x)$ and $C(x)$ in (3.3) and $\check{R}(x)$ in (3.3) solves the YBE if and only if the given BGR $S$ satisfies the identity (3.12) with $f_{3}^{ \pm}, f_{2}$ and $f_{1}^{ \pm}$corresponding to some of the cases (a), (b), and (c). We find a sufficient condition such that the given BGR satisfies the identity (3.12) is that $S$ admits the BW algebraic structure.

The general theorem of Jones [13] has already indicated that the BGR satisfying the BW algebra can be Yang-Baxterized to the solution of YBE. Our analysis coincides with Jones theorem; however, we find that for a fixed BGR $S$ having BW structure (2.1), it satisfies the identity (3.12) for the case (a) and case (b), namely $S$ is Yang-Baxterized to two types of solutions of the YBE, and both of them can be expressed in terms of the generators of the BW algebra 
[i.e., (3.16)], in particular, only one of them can be identified with Jones theorem by a suitable normalization. The reason of the "one to two" correspondence is in the sense that the BW algebra admits an obvious invariant transformation, but these two solutions have really the essential difference. The example is for the standard BGR of $D_{n}\left(B_{n}\right)$ (i.e., in Example 2.1), the correspondent two solutions of YBE are Jimbo's solutions [5] associated with $D_{n}^{(1)}\left(B_{n}^{(1)}\right)$ and the Kac-Moody algebra $A_{2 n-1}^{(2)}\left(A_{2 n}^{(2)}\right)$ respectively.

It is worth notice that there exists the concrete example that the given BGR has no BW structure but satisfies the identity (3.12) corresponding to at least one of case (a) and case (b) [19]. Thus this BGR also gives rise to the solution of YBE in the form of (3.3). There, presumably, exists a more general theory to prove, or classify whether a BGR satisfies the identity (3.12).

According to the prescription in Sect. 3, we find that the Yang-Baxterization preserves the crossing symmetry property, namely if the given BGR satisfies the crossing symmetry property in the sense of (3.23) and so do the Yang-Baxterized solutions but in the sense of (3.25). Another property of the solutions in (3.16) is that they have the fractional form in (3.20) and (3.21). The significance (for example, the connection with Riemann-Hilbert transformation) of this fractional form for the solutions of YBE is not clear yet.

In Sect.4, we give a prescription of rational Yang-Baxterization, namely, by using the reduced BW algebra (2.30), the rational solution of YBE can be generated in a similar way to that in the trigonometric case. In terms of (4.18), one of the trigonometric solutions in (3.16) goes to this rational solution, while the other trigonometric solution has no such a rational limit at the same time.

Since the BW algebra plays an important role in the process of YangBaxterization, in Sect.2, we give a sufficient condition to check whether a BGR admits the BW algebra and some examples which have BW algebra but the correspondent trace functions cannot be defined in the sense of [5]. This may also be interesting in the investigation of link polynomial and knot theory.

Acknowledgements. We wish to express our sincere thanks to Professor C.N. Yang for many enlightening discussions and suggestions. M.L. Ge would like to thank Profs. M. Jimbo, H.C. Lee, H.Sah, L. Takhtajan, and Y.S. Wu for valuable discussions. M.L. Ge is supported in part by NSF Grant PHY-89-8495 through ITP, SUNY at Stony Brook and Paul and Gabriella Rosenbaum Foundation. Y. Cheng is supported by the Y.T. Liu Fellowship through the Committee of Educational Exchange with China and partially by the Fok Ying-Tung Education Foundation of China..

\section{References}

1. Yang, C.N.: Phys. Rev. Lett. 19, 1312 (1967)

2. Baxter, R.J.: Exactly solved models in statistical mechanics. London: Academic Press 1982

3. Yang, C.N., Ge, M.L. (eds.) : Braid group, knot theory and statistical mechanics. Singapore: World Scientific 1989

4. For reference book, see: Yang-Baxter equation in integrable systems. Jimbo, M. (ed.), Singapore: World Scientific 1990

5. Jimbo, M.: Commun. Math. Phys. 102, 537 (1986), and in [3], pp. 111-134

6. Belavin, A.A., Drinfeld, V.G.: Funct. Anal. Appl. 16, 159 (1982)

7. Turaev, V.G.: Invent. Math. 92, 527 (1988)

8. Wadati, M., Deguchi, T., Akutsu, Y.: Phys. Rep. 180, 247 (1989)

9. Yu Reshetikhin, N.: Preprint LOMI, E-4-87, E-14-87

10. Ge, M.L., Wang, L.Y., Xue, K., Wu, Y.S.: Inter. J. Mod. Phys. 4, 3351 (1989)

Ge, M.L., Li, Y.Q., Xue, K.: J. Phys. 23A, 605, 619 (1990) 
11. Couture, M., Cheng, Y., Ge, M.L., Xue, K.: Preprint ITP-SB-90-05

Lee, H.C., Couture, M., Schmeing, N.C.: Preprint CRNL-TP-88-1125R

12. Ge, M.L., Xue, K.: Preprint ITP-SB-90-20

13. Jones, V.: Commun. Math. Phys. 125, 459 (1989)

14. Ge, M.L., Wu, Y.S., Xue, K.: Preprint, ITP-SB-90-02. Inter. J. Mod. Phys. (to appear)

15. Birman, J., Wenzl, H.: Trans. A.M.S. 313, 249 (1989)

Murakami, J.: Osaka J. Math. 24, 745 (1987)

16. Takhtajan, L.: Introduction to quantum group. Nankai Mathematical Physics Lectures. Singapore: World Scientific 1990 (in press)

17. Lee, H.C.: Preprint, CRNL-TP-90

18. Wenzl, H.: Ann. Math. 128, 179 (1988)

19. Ge, M.L., Gwa, L.H., Zhao, H.K.: Preprint ITP-SB-90-09

20. Yang, C.N.: Phys. Rev. 168, 1920 (1968)

21. Zamolodchikov, A.B., Zamolodchikov, Al. B.: Ann. Phys. 120, 253 (1979)

Communicated by N. Yu. Reshetikhin 